\title{
The hunt for lepton flavor violation with the Mu3e experiment
}

\author{
Sebastian Dittmeier* on behalf of the Mu3e collaboration \\ Physikalisches Institut, Ruprecht-Karls-Universität Heidelberg, \\ Im Neuenheimer Feld 226, 69120 Heidelberg, Germany \\ E-mail: dittmeier@physi.uni-heidelberg.de
}

\begin{abstract}
The Mu3e experiment will search for the charged lepton flavor violating decay $\mu^{+} \rightarrow e^{+} e^{-} e^{+}$with a targeted branching ratio sensitivity of $10^{-16}$. The sensitivity goal drives the experimental design: To distinguish the neutrinoless signal decay from background processes, excellent momentum, vertex and time resolutions of the detector system are required. An ultra-thin silicon pixel tracking detector will be constructed, complemented by a scintillating fiber and a scintillating tile detector which add precise time information to the tracks. To conduct the experiment within a reasonable time, the detector will have to cope with electrons and positrons originating from muon decays at rates up to $10^{9}$ per second. The trigger-less readout system collects the detector data, reconstructs tracks and selects events online for offline analysis. This paper describes the current status of design and construction of the Mu3e experiment and emphasizes the progress made in the development of the readout system.
\end{abstract}

The 21st international workshop on neutrinos from accelerators (NuFact2019)

August 26 - August 31, 2019

Daegu, Korea

${ }^{*}$ Speaker. 


\section{Experimental Concept}

The Mu3e experiment [1] is aiming to increase the sensitivity for the decay $\mu^{+} \rightarrow e^{+} e^{-} e^{+}$to branching ratios of the order of $10^{-16}$, an improvement by four orders of magnitude compared to the limit set by the SINDRUM experiment [2]. It will be conducted at the Paul-Scherrer-Institute in two phases. In Phase I, the currently existing $\pi \mathrm{E} 5$ muon beam line that provides up to $10^{8}$ muons per second allows to achieve a single event sensitivity of $2 \times 10^{-15}$ in 300 days of data taking. The ultimate sensitivity goal of $10^{-16}$ is foreseen to be reached in Phase II, which requires a higher intensity muon beamline ( $>10^{9}$ muons per second), which is currently under study.

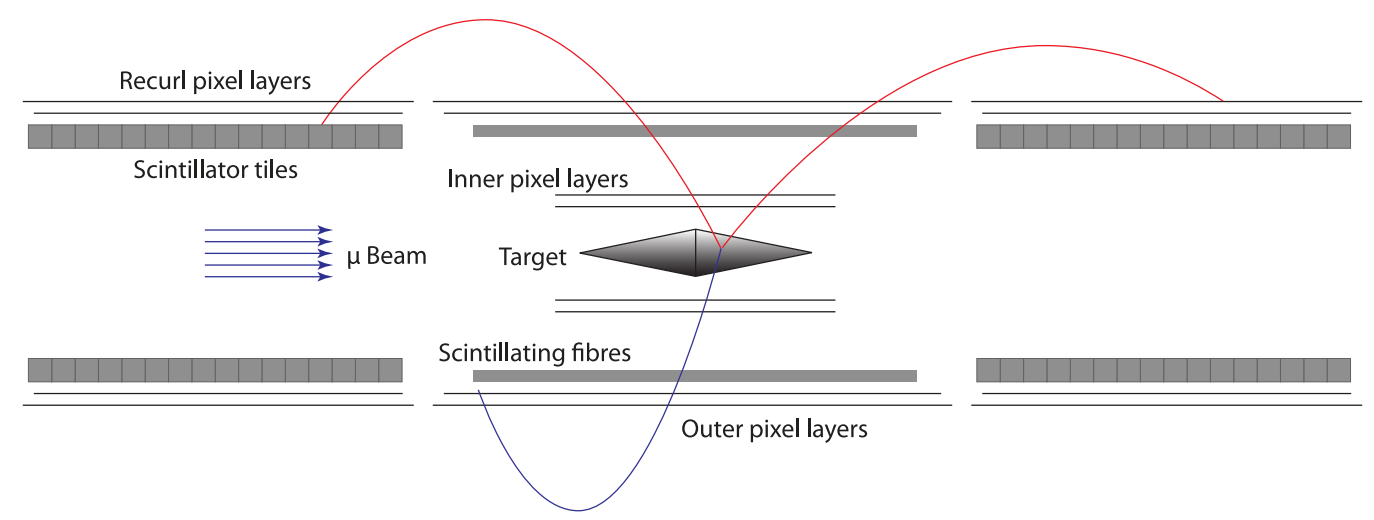

Figure 1: Transverse view of the Phase I Mu3e detector concept.

The muons are stopped to decay on a hollow double-cone shaped target within a solenoidal magnetic field of $1 \mathrm{~T}$. The target is surrounded by four silicon pixel tracking detector layers arranged in a barrel-type geometry. In-between the inner and outer two layers, three layers of scintillating fibers provide precise time measurements. Up- and downstream of the central barrel, copies of the outer pixel layers increase the acceptance for particles curling back towards the central beam axis, enhancing the momentum resolution for these tracks substantially. Underneath, scintillat-

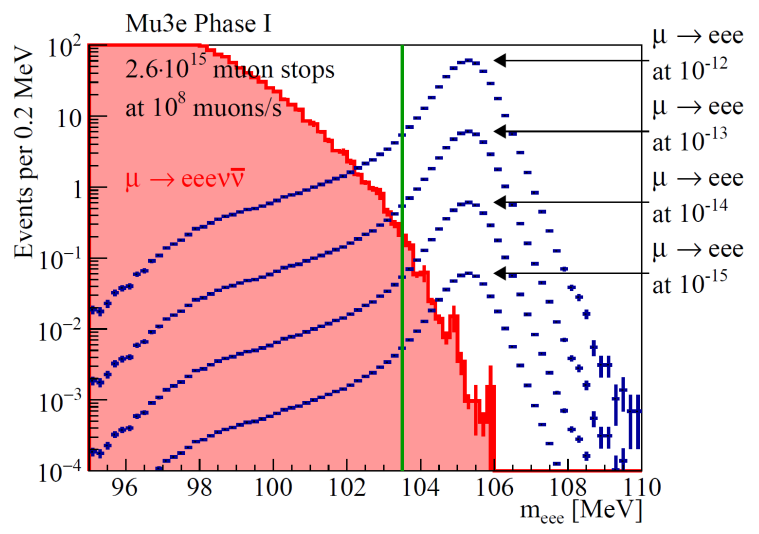

Figure 2: Simulated performance of the Mu3e experiment Phase I [3]. The invariant mass spectrum of reconstructed signal candidates is shown. The dominant background contribution is shown in red. The blue curves show the expected signal shape for different branching ratios. 
ing tiles provide an even more precise time measurement. The detector concept is illustrated in Figure 1.

The signal decay has a unique experimental signature, consisting of three coincident tracks originating from a common vertex, that distinguishes it from backgrounds. However, to reach the ultimate target sensitivity, extremely good momentum $\left(\sigma_{p}<1.0 \mathrm{MeV}\right)$, vertex $\left(\sigma_{x}<300 \mu \mathrm{m}\right)$ and time resolutions $\left(\sigma_{t}<500 / 100 \mathrm{ps}\right.$ (fiber/tile)) are required to suppress the main sources of background: the decay $\mu^{+} \rightarrow e^{+} e^{-} e^{+} v_{e} \bar{v}_{\mu}$ and combinatorial background due to the high muon decay rate. Detailed simulation studies have been carried out to evaluate the performance of Mu3e in Phase I. Figure 2 shows the reconstructed invariant mass spectrum of signal candidate events, where a signal would appear as a bump at the muon mass.

The Mu3e collaboration is currently focusing on the integration of all detector components into a complete system. To that end, very detailed CAD models have been designed and mechanical prototypes have been built for all sub-detectors. In the following, the status of the sub-detectors and the readout system is presented.

\section{Pixel Tracking Detector}

The tracking detector consists of ultra-thin layers [4] that are made of High-Voltage Monolithic Active Pixel Sensors (HV-MAPS), called MuPix, and flexible polyimide-aluminium high density interconnects. Extensive sensor research and characterization has led to the development of the MuPix8, the first large HV-MAPS, which was found to fulfill all experimental requirements [5], being more than $99.9 \%$ efficient at noise rates below $1 \mathrm{~Hz}$ per pixel [6] and offering a time resolution of about $6.5 \mathrm{~ns}$ after applying corrections for on-chip delays and time-walk [7]. Current efforts are focusing on the development of the MuPix10, the close-to-final pixel chip with an active area of $2 \times 2 \mathrm{~cm}^{2}$ and a pixel size of $80 \times 80 \mu \mathrm{m}$, that will be used for module prototyping.
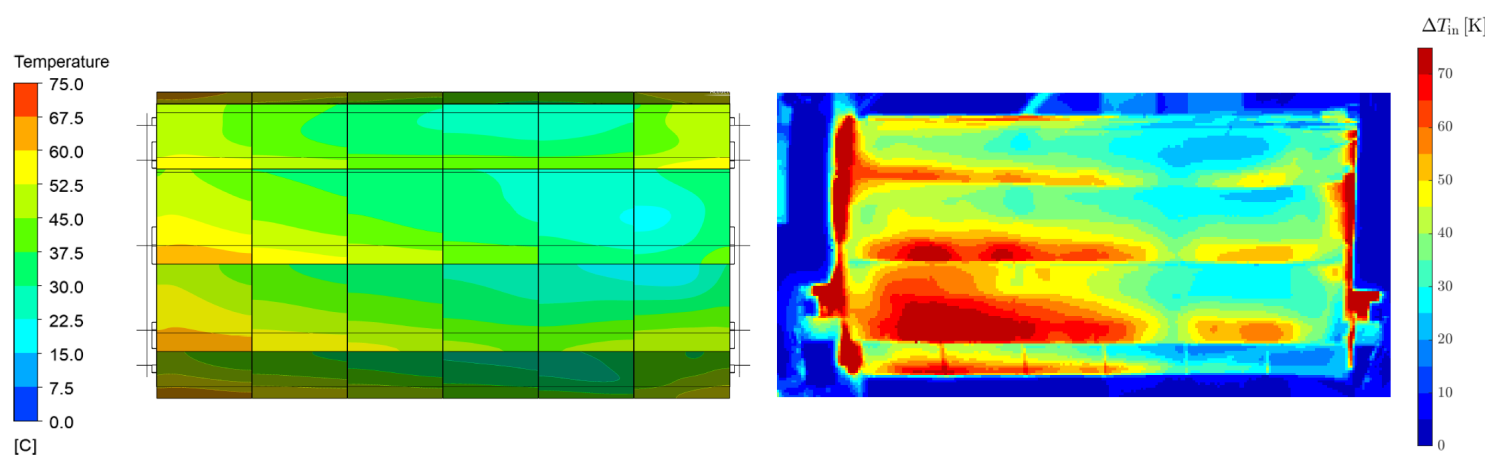

Figure 3: Comparison of helium cooling simulation studies (left) of the inner tracking detector layers with measurements (right) using a thermo-mechanical mockup being heated with an average power density of $400 \mathrm{~mW} / \mathrm{cm}^{2}$.

In order to minimize the overall material budget, the pixel sensors will be cooled with gaseous helium. Relying on Computational Fluid Dynamics (CFD) simulations, the design of the helium distribution system has been incrementally improved. To validate the simulations and also the 
mechanical design itself, a thermo-mechanical mockup of the inner two pixel layers has been built. Measurements show good agreement with simulations, as can be seen in Figure 3.

\section{Timing Detectors}

The scintillating fiber detector consists of twelve $30 \mathrm{~cm}$ long ribbons that are made of three staggered layers of $250 \mu \mathrm{m}$ thin fibers. The ribbons are coupled to silicon photomultiplier (SiPM) column arrays with 128 channels. Fiber ribbon prototypes have been produced and successfully tested [8]. In testbeam studies, the target time resolution of $500 \mathrm{ps}$ has been achieved at detection efficiencies larger than $96 \%$.

The scintillating tile detector is made of more than 6000 scintillating tiles that are individually coupled to SiPMs. A submodule prototype, combining 32 channels, has been produced and studied in testbeam measurements. A single channel time resolution of $45 \mathrm{ps}$ has been achieved, which exceeds the requirement. Both timing detectors are read out with a custom designed mixed mode ASIC, the MuTRiG, that is capable of handling rates above a MHz per channel.

\section{Readout System}

The Mu3e experiment relies on the continuous, streaming readout of zero-suppressed detector data. Custom designed front-end boards, located inside the magnet, are electrically connected to the ASICs. Each front-end board hosts an Intel Arria V FPGA that handles sensor readout and configuration, and restores the chronological order of the detector data. It transfers the data via optical links to the switching boards, which merge the data from several front-ends, inside the

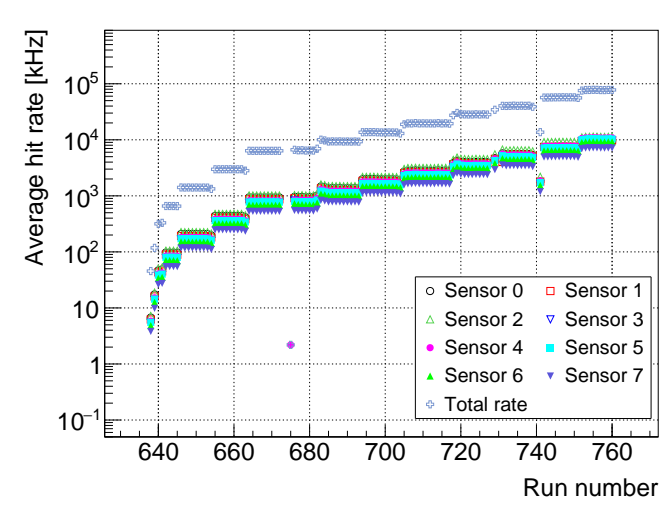

(a) Sensor hit rates during a rate scan [9]. The readout system can be successfully operated with hit rates of several $\mathrm{MHz}$ per sensor.

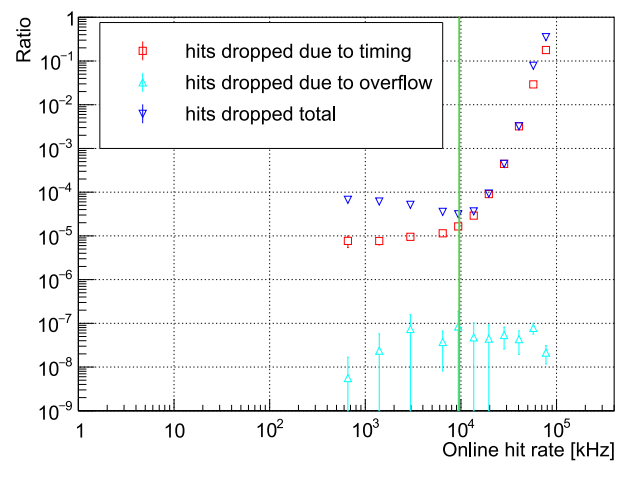

(b) Data losses in the sorting algorithm versus total system hit rates, adapted from [9]. The system can be operated without significant data losses up to the sensor occupancies expected in Mu3e Phase I (green line).

Figure 4: Readout integration study of the pixel sensors MuPix8 as 8-layer beam telescope with the front-end board prototype. 
counting house. The data is transferred to the GPU filter farm, where a dedicated fit algorithm [10] is used to reconstruct tracks. Vertex cuts are applied to identify signal candidates.

Using a prototype of the front-end board based on an Intel Stratix IV FPGA, readout integration tests with the MuPix8 pixel sensors have been conducted [9]. A beam telescope consisting of eight MuPix8 sensors has been set up and successfully operated up to hit rates of $10 \mathrm{MHz}$ per sensor, see Figure $4 \mathrm{a}$. The performance of the serial links of the pixel sensors and of the sorting algorithm [3], that restores the chronological hit order on the FPGA, has been evaluated. The electrical links could be operated stably and no impact of the hit rate on the link quality was found. The sorting algorithm performed up to the expectations. An acceptable small fraction of hits, a few times $10^{-5}$, that are read out late by the sensor are discarded in the FPGA up to hit rates of a few MHz per sensor, see Figure 4b, which is within the range of the highest expected sensors occupancies in Phase I of the Mu3e experiment.

\section{Summary and Outlook}

The Mu3e collaboration is currently focusing its work on the integration of the full detector system. A detailed mechanical design of the Mu3e experiment is available and being validated together with the helium cooling system by means of a thermo-mechanical mockup. Studies are being continued by building a mockup for the outer pixel layers. Within the year 2020, all detectors are expected to reach production readiness such that first commissioning runs can be performed with the available hardware. Readout integration studies including components from all sub-detectors are currently being carried out.

\section{References}

[1] A. Blondel et al. Research Proposal for an Experiment to Search for the Decay $\mu \rightarrow$ eee. 2013. arXiv: 1301.6113.

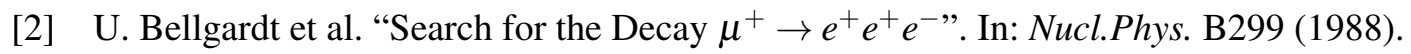

[3] A.-K. Perrevoort. "Sensitivity Studies on New Physics in the Mu3e Experiment and Development of Firmware for the Front-End of the Mu3e Pixel Detector". PhD thesis. Heidelberg University, 2018.

[4] N. Berger et al. "Ultra-low material pixel layers for the Mu3e experiment". In: JINST 11 C12006 (2016).

[5] H. Augustin et al. "Performance of the large scale HV-CMOS pixel sensor MuPix8". In: JINST 14 C10011 (2019).

[6] L. Huth. "A High Rate Testbeam Data Acquisition System and Characterization of High Voltage Monolithic Active Pixel Sensors". PhD thesis. Heidelberg University, 2018.

[7] J. Hammerich. "Analog Characterization and Time Resolution of a large scale HV-MAPS Prototype”. Master Thesis. Heidelberg University, 2018.

[8] A. Bravar. “The Mu3e Experiment at PSI”. In: SciPost Phys. Proc. (1 2019), p. 37. 
[9] S. Dittmeier. "Fast data acquisition for silicon tracking detectors at high rates". PhD thesis. Heidelberg University, 2018.

[10] N. Berger et al. "A new three-dimensional track fit with multiple scattering". In: Nucl. Instrum. Meth. A844 (2017). 